\title{
A method for the risk analysis of energy supply in Integrated Energy Systems
}

\author{
Huai Su \\ National Engineering Laboratory for Pipeline Safety/ MOE Key Laboratory of Petroleum Engineering /Beijing \\ Key Laboratory of Urban Oil and Gas Distribution Technology, China University of Petroleum-Beijing, 102249, \\ Beijing, China. E-mail: suhuai1990@163.com \\ Enrico Zio \\ Dipartimento di Energia, Politecnico di Milano, Via La Masa 34, 20156, Milano, Italy. \\ MINES ParisTech, PSL Research University, CRC, Sophia Antipolis, France. \\ E-mail: enrico.zio@polimi.it \\ Jinjun Zhang* \\ National Engineering Laboratory for Pipeline Safety/ MOE Key Laboratory of Petroleum Engineering /Beijing \\ Key Laboratory of Urban Oil and Gas Distribution Technology, China University of Petroleum-Beijing, 102249, \\ Beijing, China.E-mail: zhangij@cup.edu.cn
}

Integrated Energy Systems (IES) is critical for the sustainability in energy system development. But, the disturbances of different uncertainties and their propagation in the system make it hard to maintain a reliable service to the customers. To overcome this problem, a systematic method for the supply reliability analysis in IES is proposed in this work. In this method, the models of different units in the IES are developed considering their working mechanism and target functions. Then their random behaviours are based on their specified stochastic properties. And, their responses are integrated by a developed two-stage optimization model, to simulate the propagation of these events and to analyze the consequences in the system level. Finally, in the risk evaluation part, probabilistic and statistic descriptions are presented, and also a risk measure is adapted from Value at Risk, an important risk measure in financial analysis, for giving solid knowledge of the potential loss of service to the customers in terms of time duration, loss and probability. The effectiveness of this developed method is verified on an assumed IES. The results present its ability to give valuable information for design, extension and management of IES.

Keywords: Integrated Energy System, energy supply, risk analysis, uncertainty analysis, graph theory, optimization.

\section{Introduction}

The development of renewable energies and the threat of shortage crisis of fossil energy promote the integration of different energy resources, including traditional energy and renewable energy. Nowadays, the concepts of Integrated Energy System (IES) has wildly spread all around the world (Moslehi and Reddy, 2018). This integration is able to contribute the energy system from different perspectives, e.g., improving system flexibility and efficiency, reducing pollution and greenhouse gas emission by integrating renewable energy resources, enhancing stability of energy supply with the buffer capacity of natural gas systems ( $\mathrm{Su}$ et al., 2017), etc.

However, the interconnection between different energy systems also increases the complexity of the overall energy supply system, from the perspectives of system structure, dynamics and uncertainty. This change may bring extra risk to the security of energy supply, which means the connected energy network may be more vulnerable (Su, Zio, et al., 2018).

According to the literature survey, the energy security issue of IES has been discussed in some works. For example, Fu et at. applied a centralmoment-based method to evaluate the supply reliability of the IES, in which the modeling of the IES and the uncertainties are combined by the limit state function method and the failure probability of energy supply is estimated (Fu and Zhang, 2018). Li et at. used a method to analyze the reliability of IES, in which the uncertain behaviors of the units of IES is modeled by stochastic processes (Li et al., 2016). These works successfully analyze the reliability of the IES from specific points such as the performance of the natural gas pipelines, the effects of renewable energy productions and so on. But, actually, there are different kinds of uncertainties, 
including random failures, uncertain demand fluctuations, intermittence in renewable energy production, etc. These uncertainties may differently affect the system operation and cause different consequences. Besides, as a large, complex system, the influence of these uncertainties can propagate in the system through the complicated connection structure and system dynamic behaviors (Kuznetsova et al., 2014).

To overcome these problems, many efforts have been performed from different perspectives. Firstly, the system complexity is analyzed by considering system structure and dynamic behavior. The system has a high level of structural complexity because of its complicated topology structure, the coupling design and the heterogeneity of the components in the IES (Zio, 2016). To analyze the effects of the structure complexity, the capacity of graph theory and network flow theory have been explored (Fang, Pedroni and Zio, 2015). Besides, the dynamic properties that arise in the operation behaviors make it difficult to analyze the potential consequences of scenarios with uncertain events. Some models have been developed to simulate the complex behaviors in the energy systems, such as agent-based modeling (Ouyang, 2014), game theory (Aliabadi, Kaya and Şahin, 2017) and optimization methods (Su et al., 2019). But there is still not an effective manner to integrate multiple uncertainties, different work mechanisms of the sub-systems and system dynamics in a systematic framework. This, in some extent, is stopping us achieve a comprehensive knowledge of the ability of the IES to serve the demands.

In this paper, a systematic framework for the analysis of the risk of energy supply in IES is proposed. The proposed framework is able to analyze the ability of IES, under random unit failures, uncertain renewable energy output and uncertainties of energy demands. The models of different units in the IES, including electric transmission lines, natural gas pipelines, photovoltaic power generators, wind farms, gasfired generators and demands, are developed based the properties. The Markov process and Monte-Carlo method (Zio, 2013) are adopted to simulate the random behaviors of these units. The IES is modeled as a flow network, and a two-stage optimization method is proposed, to calculate the supply capacity of IES. Finally, the simulation results are used as the inputs of the risk analysis. In the risk evaluation part, probabilistic and statistic descriptions are presented, and also a risk measure is adapted from Value at Risk, an important risk measure in financial analysis, for giving solid knowledge of the potential loss of service to the customers in terms of time duration, loss and probability.

\section{Modelling for the units in IES}

The transportation capacities of the natural gas pipelines determine the supply capacity of the gas network system, which can further affects the risk of supply in the IES. The transportation capacities of pipelines are calculated in Eqs. 1-2.

$$
\begin{gathered}
Q_{i j}=\sqrt{\frac{\Delta P_{\max }^{2}}{K_{i j}}}(1) \\
K_{i j}=4.5399 \times 10^{8} \frac{f g Z T}{D^{5}}\left(\frac{P_{n}}{T_{n}}\right)^{2} L
\end{gathered}
$$

where $D$ is the pipeline diameter; $L$ is the pipeline length; $T$ is the Temperature of the gas; $T_{n}$ is the standard temperature condition; $f$ is Darcy's friction factor; $g$ is the gravitational acceleration; $Z$ is the gas compressibility.

The wind energy production, depending on the wind speed, can be calculated by the Eq. 3 :

$$
P_{\text {wind }}=\left\{\begin{array}{l}
P_{\text {rated }} \quad V_{\text {rated }} \leq V_{w}<V_{\text {cut_out }} \\
P_{\text {rated }} \frac{V_{w}-V_{\text {cut_in }}}{V_{\text {rated }}-V_{\text {cut_in }}} \quad V_{\text {cut_in }} \leq V_{w}<V_{\text {rated }} \\
\begin{array}{lll}
0 & V_{w}<V_{\text {cut_in }} & V_{w}
\end{array} V_{\text {cut_out }}
\end{array}\right.
$$

where $P_{\text {wind }}$ denotes output power of the wind farm; $P_{\text {rated }}$ represents the rated power of the wind turbines; $V_{\text {cut in }}$ and $V_{\text {cut out }}$ are the cut-in and cut-out wind speeds, respectively. $V_{w}$ represents the real-time wind speed of the wind. Its uncertainty is relevant for the analysis of the risk in energy supply.

Besides, the wind farm, solar power is also an important renewable energy resource. The output power of a photovoltaic (PV) generator can be calculated by Eq. 4 .

$$
P_{p v}=P_{p v, \text { rated }} \eta_{p v} \frac{G}{G_{\text {rated }}}\left[1+\gamma\left(T_{c}-T_{c, \text { rated }}\right)\right] \text { (4) }
$$

where $P_{p v}$ is the real output power of the PV; $P_{p v, \text { rated }}$ is the rated capacity of the PV; $\eta_{p v}$ denotes the combined efficiency; $\gamma$ represents the temperature coefficient of power; $T_{c}$ is the current temperature; $T_{c, \text { rated }}$ is the standard test condition temperature.

Gas-fired power generators are the main demand sites in natural gas pipeline networks and the important sources of electric power. The relationship between the electric power generation and the natural gas pipeline network is always represented by the heat rate curve as in Eq. 5. 


$$
H R=\beta_{1}+\beta_{2} P_{\text {gen }}+\beta_{3} P_{\text {gen }}^{2}
$$

where $H R$ is the heat rate; $P_{\text {gen }}$ is the generated electric power. $\beta_{1}, \beta_{2}$ and $\beta_{3}$ are the efficiency factors in the energy conversion.

Besides, customers and suppliers are considered as nodes which are consuming or providing energy in the energy network. Considering the uncertain characteristics as in real energy systems, the amount of energy used and supplied by these nodes change randomly. Their random properties are modeled in Section 3.

\section{Uncertainty modeling}

Three types of uncertainties, including random failures of units, uncertain demands and uncertain renewable source energy output, are modelled in this work.

In the natural gas pipeline networks, pipelines, compressor stations, LNG terminals (Liquefied Natural Gas) and underground gas storages (UGS) are the units determining the energy supply capacity. The assumption is made that these units have three types of running conditions, i.e., normal, degraded and failed (Su, Zhang, et al., 2018). Hence, the stochastic properties of these units are described as a Markov Process, as in Fig. 1.

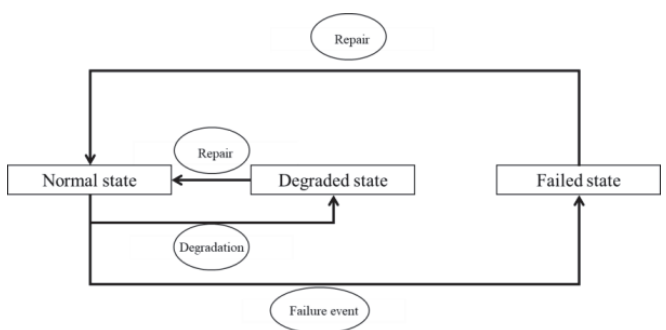

Fig. 1. Markov chain for the units considered in natural gas pipeline networks

To simulate the random changes in the generation states of the units, in the natural gas pipeline network, Monte Carlo simulation is adopted.

The details of how to use Monte Carlo simulation of the Markov process are presented in Fig. 2.

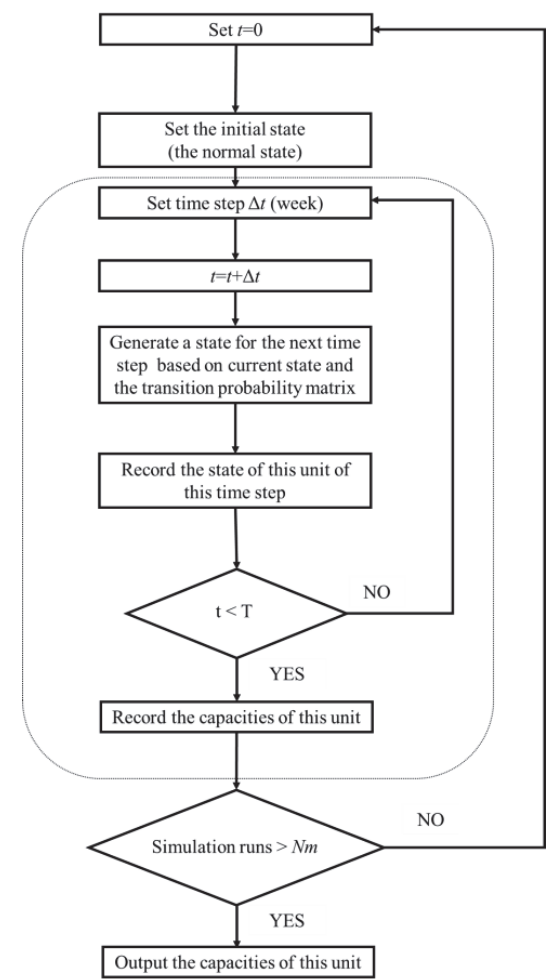

Fig. 2. The flow chart of Monte Carlo simulation for the discrete Markov process of the units in the natural gas pipeline network

As in Eq. 3, the main source of uncertainty of wind power production is the wind speed. A common description of the randomness of wind speed $V$ is the Weibull distribution, as in Eq. 6:

$$
f(V)=\left(\frac{k}{c}\right)\left(\frac{V}{c}\right)^{k-1} \exp \left[-\left(\frac{V}{c}\right)^{k}\right](6)
$$

where $k$ and $c$ are the shape and scale parameters, respectively.

The random solar radiation is most often described by a Beta distribution, whose probability density function is presented in Eq. 7:

$$
f(r)=\frac{\Gamma\left(\alpha_{p v}+\beta_{p v}\right)}{\Gamma\left(\alpha_{p v}\right) \Gamma\left(\beta_{p v}\right)}\left(\frac{G}{G_{\max }}\right)^{\alpha-1}\left(1-\frac{G}{G_{\max }}\right)^{\beta-1}
$$

where $\alpha_{p v}$ and $\beta_{p v}$ denote the parameters of the Beta distribution; $G_{\max }$ and $G$ are the maximal and actual solar radiation values; $\Gamma$ is the Gamma function.

Relevant works always estimate the probability distribution of energy demands as a normal distribution. But, the differences between the customers may significantly influence the energy supply security. Hence, the kernel estimation method (Peter D., 1985) is adopted to 
give the probability distribution models of energy demands, as in Eq. 8:

$$
f_{\text {width }_{\text {band }}}(x)=\frac{1}{n_{\text {size }} \text { width }_{\text {band }}} \sum_{i=1}^{n_{\text {sze }}} \operatorname{Ker}\left(\frac{x-x_{i}}{\text { width }_{\text {band }}}\right) \text { (8) }
$$

where $f(x)$ represents the PDF of the customer demand $x$; Ker is the kernel function; $n_{\text {xize }}$ is the size of the data set; width $_{\text {band }}$ represents the width of the kernel band.

The random outputs of the renewable power sources are sampled by Monte Carlo simulation, according to the probability distributions of the stochastic parameters in their physical models. The amount of energy demands of the customers are also sampled based on the distribution models. The running states of the electric transmission lines are simulated by nonsequential Monte Carlo sampling.

\section{Supply capacity analysis model for IES}

In this part, the sub-systems, i.e., power generators, transmission systems and customers, are considered as agents.

Firstly, the electric power demands are transmitted to the communication and control agent called Independent System Operator (ISO), which is responsible for communication between the sub-systems in the IES. The potential generator capacities of the renewable energy sources are simulated as a stochastic process according to Section 3. Then, the transmission plan in the electric power grid is optimized by the Ford-Fulkerson (FF) algorithm, to satisfy the energy demands. In this stage, the required gasfired power can be determined. The natural gas demands of the gas-fired power plants are reported to the ISO. And the plan of natural gas distribution in the pipeline network, for satisfying the customers, can be optimized based on the methods reported in Fig. 3 ( $\mathrm{Su}$ and Zio, 2018), with the supply capacities of importers, LNG terminals and UGS are reported. In some conditions, the limited resources cannot completely satisfy the required energy of the power grids. Then, the ISO needs to recalculate the real energy which can be supplied to the demand side. In this process, the energy transmission plan and outputs of the flexible energy resources are optimized. Finally, the simulation results are used as the inputs of the risk analysis.

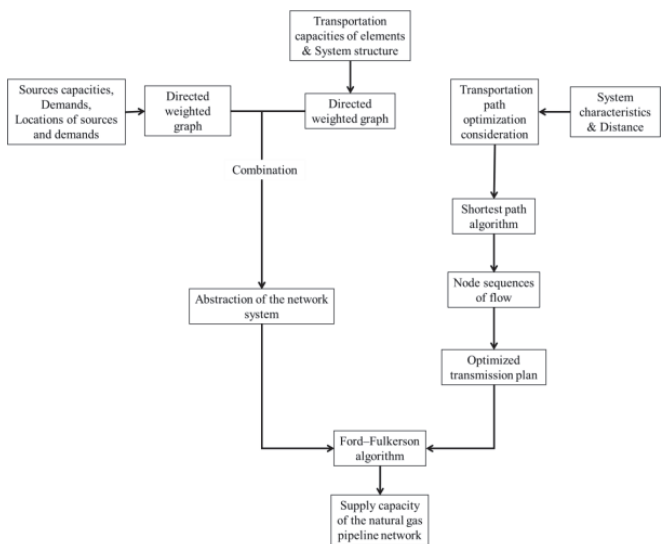

Fig. 3. Flowchart of the capacity analysis of the network systems

\section{Risk analysis method for energy supply in IES}

The risk analysis for energy supply in the IES aims to obtain an comprehensive measure of the system ability to satisfy the demands under multiple uncertainties, and quantify the potential loss. The analysis results can be used to uncertain conditions of component failures, demand changes, resource interruption and others.

Firstly, as most of the relevant works do, the statistic and probabilistic descriptions are used as the indices. Besides, to better quantify the potential loss, a risk measure is adapted for providing information on the potential loss of service to the customers in terms of probability of the loss occurrence, loss and time duration. Hence, we here introduce the concept of Value at Risk, typical used in financial portfolio risk management.

$$
\operatorname{VaR}_{p}=\inf \left\{x \mid F_{\alpha}(s) \geq p\right\}(9)
$$

in this measurement, inf is the smallest real number; $F_{\alpha}(s)$ is the CDF value of potential energy shortage $s ; p$ is the confidence level, in the range $[0,1] ; \alpha$ is the time horizon, defined as one day in the case study.

\section{Case study}

The target IES is a natural gas pipeline network (Fig. 4), and an electric power grid (Fig. 5 ) with one PV power generator and two wind farms. The two energy systems are linked by four gas-fired power plants, marked in Figs 5. The assumed natural gas pipeline network is adopted from (Praks, Kopustinskas and Masera, 2015). The electric power grid is based on the network provided in (Hu et al., 2016). 


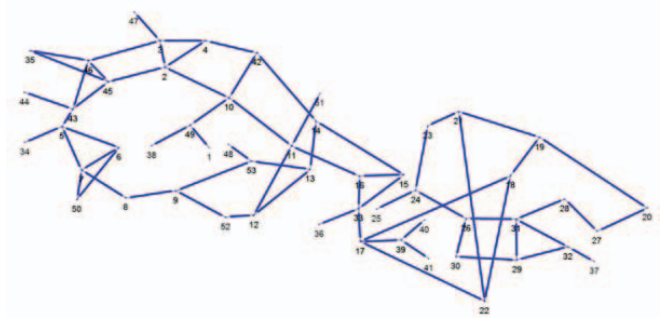

Fig. 4. Layout of the natural gas pipeline network

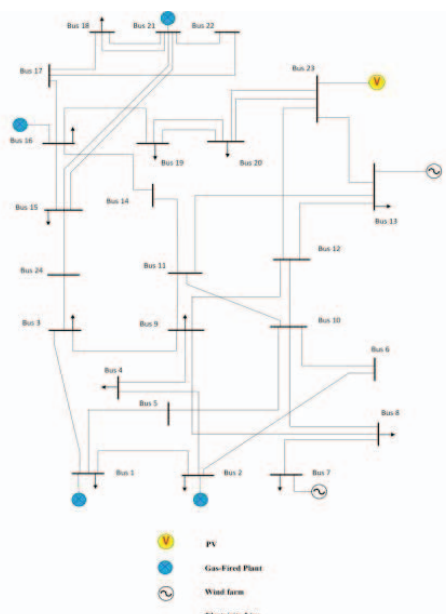

Fig. 5. Layout of the electric power grid with renewable energy productions

Firstly, the statistical method is used to calculate the global performance of supply reliability in this IES. The analysis results are able to provide helpful knowledge for system design and extension. To reasonably present the system reliability performance, the normalization, by converting the supplied energy value to the percentages in the total demands, is carried out for the statistical analysis. Because directly analysing the real amount of the supplied energy directly cannot present the time-dependent consequences under the dynamic demands. The analysis results are showed in Tables 1-2.

Table 1. Statistical analysis of the normalized supply performance of the natural gas pipeline network in the IES

\begin{tabular}{l|cccc}
\hline & Max & Min & Mean & Perf $_{75 \%}$ \\
\hline Normal & 1.000 & 0.508 & 0.987 & 1.000 \\
\hline Case A & 1.000 & 0.476 & 0.980 & 0.969 \\
\hline Case B & 0.937 & 0.498 & 0.890 & 0.891 \\
\hline Case C & 1.000 & 0.618 & 0.987 & 1.000 \\
\hline
\end{tabular}

Table 2. Statistical analysis of the global normalized supply performance of the electric power grid in the IES

\begin{tabular}{l|cccc}
\hline & Max & Min & Mean & Perf $_{75 \%}$ \\
\hline Normal & 1.000 & 0.407 & 0.982 & 0.996 \\
\hline Case A & 1.000 & 0.353 & 0.981 & 0.991 \\
\hline Case B & 0.977 & 0.269 & 0.913 & 0.914 \\
\hline Case C & 1.000 & 0.317 & 0.982 & 0.996 \\
\hline
\end{tabular}

Tables 1-2 show that the worst energy shortage (Min column) in the natural gas system is a supply reduction from 1.000 to 0.508 of the whole demand. From another perspective, the Mean column presents that the average ability of the IES to cover the demands in the natural gas system is relatively good $(0.9874$ of the total demand). Besides, the 5th column shows that the system can satisfy $99.62 \%$ of the overall demands with $75 \%$ confidence. Hence, in the normal case, the system can serve the customers to a good level, although some unexpected, highconsequence conditions may occur. Some similar conclusions can also be obtained for the electric power system, according to Table 2 .

According to the results in the Normal Case and Cases A-C, the results about the impacts of the energy resources with relatively high uncertainty can be obtained. Tables 1-2 show that the capacity reduction of the LNG terminal leads to severe degradations of the reliability performance in the natural gas system (the average performance drops from 0.9874 to 0.8898 ) and the electric power grid (the average performance drops of $7 \%$, from 0.9823 to 0.9130 ). These results indicate the importance of the LNG terminal for the supply reliability. And, the results of Cases A and B show that the IES becomes vulnerable, according to the significant drops in the 3rd quartile capacities and in the minimum capacities.

The Cumulative Distribution Functions (CDFs) of the energy are presented in Figs. 6-8. In Fig. 6, we can see that the most severe consequence is a shortage of around $12 \mathrm{MCM} / \mathrm{d}$ in the Normal Case. But in Case A, it may suffer the worst shortage, among all four cases, of about $18 \mathrm{MCM} / \mathrm{d}$. In Scenario B, with the capacity reduction of LNG $\mathrm{B}$, the IES is much more vulnerable compared with that in Scenario A, with PV capacity reduction. But, in the extreme situations, the natural gas pipeline networks in Scenario B presents better capacity to withstand the situation, than that in Scenario A. 


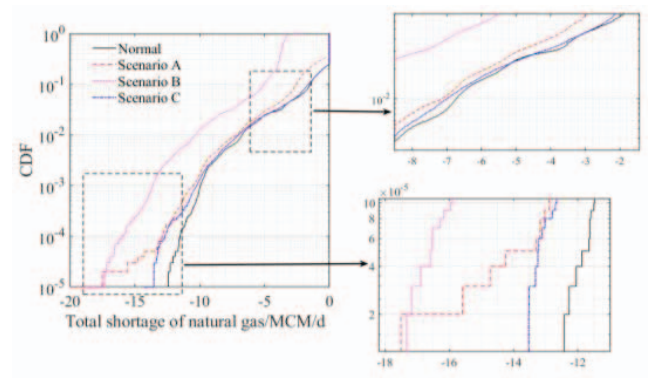

Fig. 6. CDF of the global natural gas shortage

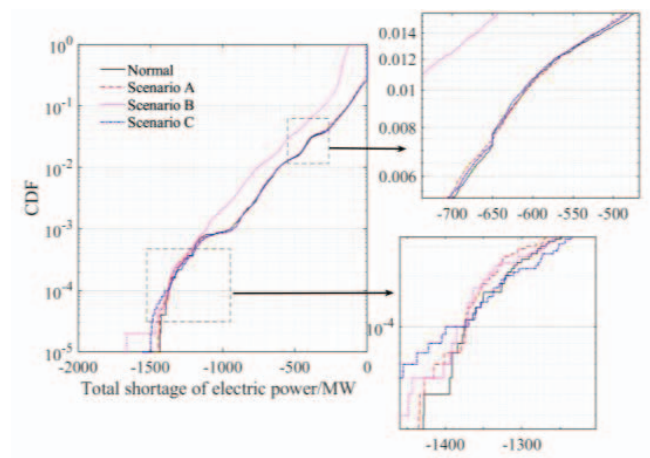

Fig. 7. CDF of the global electric power shortage

Table 3. Potential losses of services to the customers based on Value at Risk

\begin{tabular}{|c|c|c|c|c|c|c|}
\hline \multirow{2}{*}{$\begin{array}{l}\text { pe of } \\
\text { lergy }\end{array}$} & \multicolumn{2}{|c|}{ Normal } & \multicolumn{2}{|c|}{ Case A } & \multicolumn{2}{|c|}{ Case B } \\
\hline & $\mathrm{VaR}_{95}$ & $\mathrm{VaR}_{99}$ & $\mathrm{VaR}_{95}$ & $\mathrm{VaR}_{99}$ & $\mathrm{VaR}_{95}$ & $\mathrm{VaR}_{99}$ \\
\hline $\begin{array}{l}\text { atural } \\
\text { gas } \\
\mathrm{CM} / \mathrm{d})\end{array}$ & 2.74 & 6.73 & 3.52 & 7.15 & 6.42 & 10.35 \\
\hline $\begin{array}{l}\text { ectric } \\
\text { ower } \\
\text { MW) }\end{array}$ & 235.04 & 609.35 & 248.29 & 607.18 & 428.37 & 758.35 \\
\hline
\end{tabular}

From Table 3, it can be seen that, in the Normal Case, the IES may suffer a natural gas shortage of $6.73 \mathrm{MCM} / \mathrm{d}$ or more, expected on one day out of 100 days (because the probability is 0.01 ).

\section{Conclusion}

In this work, a systematic framework is developed for risk analysis of the energy supply in IES. The analysis can provide a global knowledge of the energy supply security, under uncertainties in renewable energy production, customer demand change and system failure.

The proposed framework includes three parts: uncertainty analysis, supply capacity analysis and risk analysis. Firstly, detailed stochastic models are developed for the units in IES, based on their functions and properties. Then, a two-stage optimization model is developed to integrate the outputs of the uncertain modelling and to effectively calculate the capacity of the system to serve the demands. Finally, the risk analysis method is proposed based on statistical indices and Value at Risk.

The developed method is verified on an assumed IES. And the results show that this method is able to provide solid knowledge of the potential loss of service in terms of time duration, loss and probability, and can help to analyse the reliability performance of the IES, under random unit failures, uncertain renewable energy output and uncertainties of energy demands..

\section{Acknowledgement}

This work is supported by National Natural Science Foundation of China [grant number 51904316], and the research fund provided by China University of Petroleum, Beijing [grant number 01JB20188428].

\section{References}

Aliabadi, D. E., Kaya, M. and Şahin, G. (2017) 'An agent-based simulation of power generation company behavior in electricity markets under different market-clearing mechanisms', Energy Policy, pp. 191-205. doi: 10.1016/j.enpol.2016.09.063.

Fang, Y., Pedroni, N. and Zio, E. (2015) 'Optimization of Cascade-Resilient Electrical Infrastructures and its Validation by Power Flow Modeling', Risk Analysis, 35(4), pp. 594-607. doi: 10.1111/risa.12396.

Fu, X. and Zhang, X. (2018) 'Failure probability estimation of gas supply using the central moment method in an integrated energy system', Applied Energy. Elsevier, 219(January), pp. 110. doi: 10.1016/j.apenergy.2018.03.038.

Hu, Y. et al. (2016) 'An NSGA-II based multiobjective optimization for combined gas and electricity network expansion planning', Applied Energy. Elsevier, 167, pp. 280-293. doi: 10.1016/J.APENERGY.2015.10.148.

Kuznetsova, E. et al. (2014) 'An integrated framework of agent-based modelling and robust optimization for microgrid energy management', 
Applied Energy, 129, pp. 70-88. doi:

10.1016/j.apenergy.2014.04.024.

Li, G. et al. (2016) 'Reliability evaluation of integrated energy systems based on smart agent communication', Applied Energy, 167, pp. 397406. doi: 10.1016/j.apenergy.2015.11.033.

Moslehi, S. and Reddy, T. A. (2018)

'Sustainability of integrated energy systems: A performance-based resilience assessment methodology', Applied Energy. Elsevier, 228(April), pp. 487-498. doi: 10.1016/j.apenergy.2018.06.075.

Ouyang, M. (2014) 'Review on modeling and simulation of interdependent critical infrastructure systems', Reliability Engineering and System Safety, 121, pp. 43-60. doi: 10.1016/j.ress.2013.06.040.

Peter D., H. (1985) 'Kernel estimation of a distribution function', Communications in Statistics - Theory and Methods. Marcel Dekker, Inc. , 14(3), pp. 605-620. doi: $10.1080 / 03610928508828937$.

Praks, P., Kopustinskas, V. and Masera, M. (2015) 'Probabilistic modelling of security of supply in gas networks and evaluation of new infrastructure', Reliability Engineering and System Safety. Elsevier, 144, pp. 254-264. doi: 10.1016/j.ress.2015.08.005.

Su, H. et al. (2017) 'An integrated systemic method for supply reliability assessment of natural gas pipeline networks', Applied Energy, 209(October 2017), pp. 489-501. doi: 10.1016/j.apenergy.2017.10.108.

Su, H., Zio, E., et al. (2018) 'A systematic framework of vulnerability analysis of a natural gas pipeline network', Reliability Engineering \& System Safety, 175, pp. 79-91. doi: 10.1016/j.ress.2018.03.006.

Su, H., Zhang, J., et al. (2018) 'An integrated systemic method for supply reliability assessment of natural gas pipeline networks',
Applied Energy. Elsevier, 209, pp. 489-501. doi: 10.1016/J.APENERGY.2017.10.108.

Su, H. et al. (2019) 'A systematic data-driven Demand Side Management method for smart natural gas supply systems', Energy Conversion and Management. Pergamon, 185, pp. 368-383. doi: 10.1016/J.ENCONMAN.2019.01.114.

Su, H. and Zio, E. (2018) 'A flow-based method for identifying critical pipelines in complex natural gas supply systems', pp. 2051-2057.

Zio, E. (2013) 'The Monte Carlo simulation method for system reliability and risk analysis', Springer, pp. 1-5. doi: 10.1007/978-1-44714588-2.

Zio, E. (2016) 'Challenges in the vulnerability and risk analysis of critical infrastructures', Reliability Engineering and System Safety. Elsevier, 152, pp. 137-150. doi: 10.1016/j.ress.2016.02.009. 